\title{
Recent results from heavy flavour physics on the lattice
}

\author{
Michele Della Morte* \\ Institut für Kernphysik and Helmholtz Institut, University of Mainz, \\ Johann-Joachim-Becher Weg 45, D-55099 Mainz, Germany \\ E-mail: morte@kph.uni-mainz.de
}

\begin{abstract}
We discuss some recent lattice results on a few selected hadronic quantities relevant for heavy flavour phenomenology and present some recent theoretical developments. We put the emphasis on the challenges, which have to be faced, on the way to precise heavy flavour physics from the lattice. We also discuss the importance in the search for New Physics of possible future lattice studies of the form factors entering $B \rightarrow K$ and $B \rightarrow K^{*}$ semileptonic decays.
\end{abstract}

35th International Conference of High Energy Physics - ICHEP2010,

July 22-28, 2010

Paris France

* Speaker. 


\section{Heavy quarks on the lattice}

Heavy flavour phenomenology is an important tool for the indirect search of New Physics (NP) and for constraining possible extensions of the Standard Model (SM). As these indirect NP effects are expected to be small [1], it is absolutely necessary to have all systematics affecting the theoretical predictions under control. In this framework the lattice can provide first principle determinations of important quantities such as heavy mesons decay constants and form factors for rare B-decays. Cutoff effects, i.e. the dependence of the results on the lattice resolution, are one of the main systematics in this case. It is clear that in order to properly describe the propagation of heavy quarks fine lattice resolutions are needed. The issue has been quantitatively studied in [2], by computing the decay constant $F_{\mathrm{D}_{\mathrm{s}}}$ of the $D_{\mathrm{s}}$ meson, in the quenched approximation, over a large range of lattice spacings $a$, namely $0.03 \mathrm{fm} \lesssim a \lesssim 0.09 \mathrm{fm}$. The results are shown in fig. 1 . As

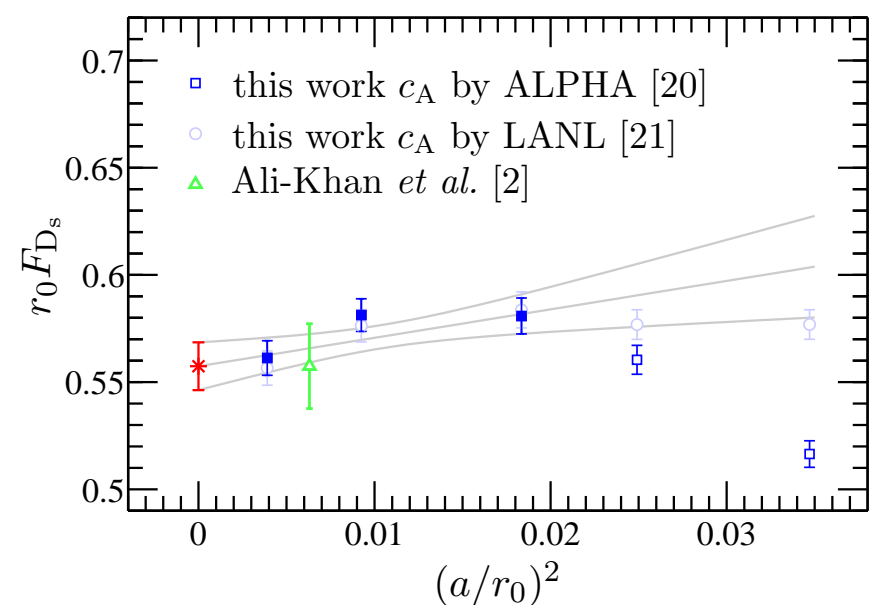

Figure 1: The (quenched) decay constant $F_{\mathrm{D}_{\mathrm{s}}}$ vs the lattice spacing for the $\mathrm{O}(a)$ improved Wilson-Clover action and two different choices of the improved current. The lattice spacing is measured in units of the scale $r_{0} \simeq 0.5 \mathrm{fm}$ [3]. Plot from [2].

the action and the operators are both $\mathrm{O}(a)$ improved, scaling violations are expected to be linear in $a^{2}$. However such a behaviour sets in for small lattice resolutions only, $a \lesssim 0.07 \mathrm{fm}$ (i.e. the three finest resolutions in the plot). If results for $a \gtrsim 0.07 \mathrm{fm}$ only had been available then the continuum extrapolation would have produced a rather different number for $F_{\mathrm{D}_{\mathrm{s}}}$. This despite the fact that the data show a linear dependence on $a^{2}$ in that region of lattice spacings. A second set of numbers is obtained by adopting another definition of the improved axial current (grey symbols in the figure), which differs from the first one by $\mathrm{O}\left(a^{2}\right)$. The cutoff effects appear to be smaller for this second definition, however the situation is reversed for other observables as the charm quark mass, as discussed in [2]. It is clear anyway that by considering two different $\mathrm{O}(a)$ improved regularizations of the current some indications can be gathered on the onset of the scaling region. The overall lesson seems to be that Symanzik improvement programme works also for heavy quarks, but lattice spacings smaller than $0.08 \mathrm{fm}$ are needed.

A second example on how delicate continuum limit extrapolations are for quantities involving heavy quarks is obtained by revisiting the so called $F_{\mathrm{D}_{\mathrm{s}}}$ tension. The extremely precise result $F_{\mathrm{D}_{\mathrm{s}}}=241$ (3) MeV obtained in 2007 by the HPQCD Collaboration [4] using $2+1$ flavours of rooted staggered quarks turned out to differ by more than three standard deviations from the experimental 
estimate $F_{\mathrm{D}_{\mathrm{s}}}=274(11) \mathrm{MeV}$ (see [5]), produced by CLEO-c by analyzing tauonic $D_{\mathrm{s}}$ decays [6]. Now both numbers have moved by one or two sigma. The result from CLEO-c with updated statistics is $F_{\mathrm{D}_{\mathrm{s}}}=259(6)(3) \mathrm{MeV}$ [7]. The HPQCD Collaboration has updated the 2007 result by incorporating a new and more accurate determination of the lattice spacing and by including results at finer lattice resolutions, covering the range $0.044 \mathrm{fm} \leq a \leq 0.15 \mathrm{fm}$ (to be compared with the range $0.09 \mathrm{fm} \leq a \leq 0.15 \mathrm{fm}$ used in [4]). This yields the value $F_{\mathrm{D}_{\mathrm{s}}}=248.0(25) \mathrm{MeV}$ [8]. The tension has solved and the discrepancy is below 1.5 combined standard deviations.

Having emphasized the importance of simulating at very fine lattice spacings to study heavy flavour physics on the lattice, let us briefly discuss some algorithmic difficulties in approaching the continuum limit. Critical slowing down of Monte Carlo algorithms is expected near phase transitions, as the one which defines the continuum limit. In [9] it was reported that a critical exponent as large as 5 can be measured in typical Monte Carlo simulations, for quantities like the topological charge, this implies a scaling as $a^{-10}$ for full QCD simulations. It was on the other hand also observed that other quantities such as heavy-light pseudoscalar correlation functions couple only weakly to the slow modes affecting the topological charge. The possibility however remains that modes even slower than those detected in that study may be present. A robust statistical error estimate is therefore a delicate issue and improved estimators have been proposed in [9]. More generally critical slowing down provides a reason to clearly prefer few but long Monte Carlo chains over several short ones.

The situation is different for B-physics on the lattice. In this case the b-quark is treated nonrelativistically and coarser lattice resolutions than those considered for D-physics can be used. An attractive option in this respect is Heavy Quark effective Theory (HQET) [10] on the lattice. HQET provides the correct asymptotic description of QCD correlation functions in the limit $m_{\mathrm{b}} \rightarrow \infty$. Subleading effects are described by higher dimensional operators whose coupling constants are formally $\mathrm{O}\left(1 / m_{\mathrm{b}}\right)$ to the appropriate power. Due to the appearance of power divergent mixings (as $a$ goes to 0 ), the theory must be formulated and renormalized non-perturbatively already at the leading (static) order [11]. A non-perturbative treatment of HQET on the lattice including $1 / m_{\mathrm{b}}$ corrections has been recently put forward in $[12,13,14]$ with first applications on the determination of the b-quark mass, the mesonic spectrum in the $B_{\mathrm{s}}$ meson sector and the $B_{\mathrm{s}}$ decay constant. These studies have been performed in the quenched approximation but their extension to the $N_{\mathrm{f}}=2$ dynamical case is almost completed and preliminary results are already available [15]

\section{2. $B \rightarrow K$ and $B \rightarrow K^{*}$ semileptonic decays}

These rare decays are interesting potential probes of Physics beyond the Standard Model since NP diagrams could contribute at the same level as SM ones. The branching ratios are measured at Babar, Belle and $\mathrm{CDF}$ at $\mathrm{O}\left(10^{-7}-10^{-6}\right)$ [17], consistent with the SM. These modes are also among the key measurements in the LHCb physics programme [16]. Beyond the rate, several observables can be obtained which are sensitive to different electroweak couplings. On the theory side most of the studies concentrated on the form factors in the large recoil region, where QCD factorization applies [18]. However, as pointed out in [19], the low recoil region is equally relevant in constraining NP. This offers the possibility for the lattice to contribute, as the low recoil region is the one reliably accessible to lattice calculations. As of today only preliminary results from one study using moving-NRQCD exist [20]. This is a very difficult calculation as seven different form 
factors need to be computed for the decay $B \rightarrow K^{*} l^{+} l^{-}$. The confidence on lattice results for these form factors will improve in the future as different approaches are considered .

\section{Conclusions}

The precision of lattice computations of hadronic parameters related to heavy-flavour physics has considerably increased in the last years, as it is required for the approach to be a tool for search of New Physics. As the accuracy improves, systematic effects previously considered to be negligible become relevant. Such calculations therefore remain very challenging and new ideas and techniques are constantly being developed to keep the systematics under control. In some cases this activity even leads to a deeper uderstaning of fundamental properties of QCD and gauge theories [21].

At the same time new processes are being considered for signals of New Physics. The lattice can play an important role there in providing a first-principle determination of the relevant form factors and matrix elements within the Standard Model.

Acknowledgements. I wish to thank all the members of ALPHA and in particular Rainer Sommer for the pleasant collaboration. I am grateful to Gudrun Hiller for useful discussions.

\section{References}

[1] M. Artuso et al., Eur. Phys. J. C57 (309) 2008.

[2] J. Heitger and A. Jüttner, JHEP 0905 (2009) 101.

[3] R. Sommer, Nucl. Phys. B411 (1994) 839.

[4] E. Follana, C. T. H. Davies, G. P. Lepage and J. Shigemitsu, Phys. Rev. Lett. 100 (2008) 062002.

[5] M. Della Morte, PoS LAT2007 (2007) 008.

[6] K. M. Ecklund et al., CLEO Collaboration, Phys. Rev. Lett. 100 (2008) 161801.

[7] P. Naik et al., CLEO Collaboration, Phys. Rev. D80 (2009) 112004.

[8] C. T. H. Davies et al., HPQCD Collaboration, arXiv:1008.4018 [hep-lat].

[9] S. Schaefer, R. Sommer and F. Virotta, arXiv:1009.5228 [hep-lat].

[10] E. Eichten and B. Hill, Phys. Lett. B234 (1990) 511.

[11] J. Heitger and R. Sommer, JHEP 0402 (2004) 022.

[12] B. Blossier, M. Della Morte, N. Garron and R. Sommer, JHEP 1006:002, 2010.

[13] B. Blossier et al., ALPHA Collaboration, JHEP 1005:074, 2010.

[14] B. Blossier et al., ALPHA Collaboration, arXiv 1006.5816 [hep-lat].

[15] B. Blossier et al., ALPHA Collaboration, PoS (Lattice 2010) 308.

[16] P. Perret, LHCb Collaboration, arXiv:0901.2856 [hep-ex].

[17] E. Barberio et al.[Heavy Flavour Averaging Group], arXiv:0808.1297 [hep-ex].

[18] M. Beneke, T. Feldmann and D. Seidel, Nucl. Phys. B 612 (2001) 25.

[19] C. Bobeth, G. Hiller, D. van Dyk, JHEP 1007 (2010) 098.

[20] Z. Liu et al, PoS LAT2009 (2009) 242.

[21] M. Lüscher, JHEP 1008 (2010) 071. 